\title{
Efectos de altos niveles de proteína en el último tercio de la gestación sobre el crecimiento postnatal de la progenie en bovinos para carne
}

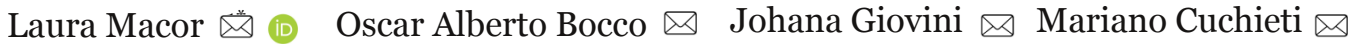 \\ Maria Valeria Coniglio $ه$ María Eugenia Ortiz $ه$ \\ Universidad Nacional de Río Cuarto, Argentina
}

\section{High protein level effects during last gestation in beef cattle in offspring postnatal growth}

\begin{abstract}
Lows prepartum nutritional levels in breeding cows has been linked to lower progeny productivity through fetal programming mechanism. Few studies have been done on the impact of a protein excess during late gestation on the afore mentioned mechanism. This situation can be observed in systems that produce genetic material. The objective of this research was to assess the effect of a high protein level pastoral diet during the last third of gestation on the progeny growth. Sixteen Aberdeen Angus multiparous pregnant females were fed diets with two different protein levels during the last third of gestation: one a degraded pasture (Baccharis coridifolia, Baccharis articulata, Spartina sp., Sporobolus sp., Stipa sp. and Setaria leucopila) (CP 13.92 \% and Dig 65.5 \%) and the other a triticale pasture (CP $18.2 \%$ and Dig $68.7 \%$ ). The CP inputs were $10 \%$ and $59 \%$ higher than daily requirements, respectively. A completely randomized experimental design with two replications was used and 8 animals were allocated to each treatment. Birth weight, weight at 205 days, pre-weaning daily weight gain and yearling weight were evaluated. Higher protein intake negatively affected growth potential $(\mathrm{p}<0.05)$, without affecting birth weight. The high levels of CP, mainly being degradable protein in rumen, could generate a greater metabolic demand on the pregnant cow due to an excessive ureagenesis, which competes with other metabolic processes for metabolic energy and different metabolites, including methionine, an amino acid associated to the fetal programming mechanism.
\end{abstract}

Key words: beef cattle, protein level, Tritico secale

Resumen. Un bajo nivel nutricional preparto de la vaca de cría, se ha relacionado con menor productividad en la progenie a través del mecanismo de programación fetal. El exceso de proteína en la gestación tardía sobre este mecanismo es aún, poco estudiado. Esta situación puede observarse en sistemas dedicados a la venta de material genético (cabañas núcleo o multiplicadores). El objetivo del trabajo fue evaluar el efecto de una dieta pastoril de elevado nivel proteico (59 \% por encima de los requerimientos) durante el último tercio de la gestación sobre el crecimiento de la progenie. Se utilizaron 16 hembras preñadas multíparas Aberdeen Angus alimentadas con diferentes niveles proteicos durante el último tercio de la gestación: uno sobre una pastura degradada compuesta principalmente por mío-mío (Baccharis coridifolia) y carqueja (Baccharis articulata), espartillo (Spartina sp.), pasto alambre (Sporobolus sp.), flechilla (Stipa sp.) y cola de zorro (Setaria leucopila) y el otro sobre triticale (Tritico secale). La Proteína Bruta (PB) fue de (PB $13.92 \%$ y $18.2 \%$ respectivamente y el \% de Digestibilidad in vitro fue de $65.5 \%$ y $68.7 \%$ ). Los aportes de PB fueron $10 \%$ y $59 \%$ superiores a los requerimientos diarios, respectivamente. Se empleó un diseño experimental completamente aleatorizado con dos repeticiones y se asignaron al azar 8 animales a cada tratamiento. Se evaluó peso al nacer, peso a los 205 días, aumento diario de peso vivo predestete y peso al año. Mayores aportes de proteína afectaron negativamente el potencial de crecimiento (p < 0.05), sin afectar el peso al nacer. Los altos niveles de proteína bruta pudieron generar mayor demanda metabólica sobre la vaca gestante por efecto de la ureagénesis excesiva, que compite con otros procesos metabólicos por energía metabólica y diferentes metabolitos, entre ellos la metionina, aminoácido asociado al mecanismo de programación fetal.

Palabras Clave: bovinos para carne, alto nivel proteico, Tritico secale

Recibido: 2019-02-19. Aceptado: 2020-09-30

*Autor para la correspondencia: Imacor@ayv.unrc.edu.ar 


\section{Introducción}

Se ha determinado que el nivel proteico en dietas preparto, puede afectar la productividad del sistema de cría, la eficiencia del crecimiento en los terneros y la reproducción futura de la reposición (Martin et al., 2007; Radunz et al., 2009; Du et al., 2009; Larson, 2009; Funston et al., 2010; Underwood et al., 2010; Reynolds y Caton, 2012; Scheffler et al., 2014.). Estos efectos se atribuyen al proceso de programación fetal; mecanismo definido como la respuesta a un evento específico ocurrido durante un período crítico del desarrollo fetal, que altera su trayectoria de manera cualitativa, cuantitativa o ambas, y produce efectos persistentes en la vida del animal (Barker et al.,1992; 2004; Nathanielsz et al., 2007). Una reducción del número de fibras musculares durante el período de miogénesis primaria o secundaria a causa de una subnutrición materna, principalmente proteica, genera consecuencias irreversibles en la descendencia (Du et al., 2010). Respecto al efecto del exceso de proteínas en la gestación tardía, existe aún poco conocimiento, principalmente sobre proteína degradable en rumen. Wilson et al. (2015) y Radunz et al. (2010) utilizaron proteína no degradable en rumen en la formulación de las dietas preparto y no observaron diferencias en el potencial de crecimiento pre o post destete de la progenie (Gicquel et al., 2008; Du et al., 2009; Du et al., 2010; Lan et al., 2013).

La mal nutrición y otros factores adversos de la madre afectarían la expresión de los genes, modulando de forma negativa, diferentes aspectos del desarrollo fetal, que dependen del momento de la preñez en que ocurre la restricción. Reed y Govoni (2017) identificaron, además, modificaciones en las funciones de células madres en la descendencia como resultado de una pobre nutrición materna.

\section{Materiales y Métodos}

\section{Animales}

Se utilizaron bovinos hembras multíparas de raza Aberdeen Angus de entre 5 a 6 años de vida, con un peso promedio de $400 \mathrm{~kg}$ y una condición corporal de 2.5. Se trabajó con un grupo 16 hembras gestantes, que en 2017 parieron 16 terneros, 10 machos y 6 hembras.

\section{Condiciones Agroecológicas}

El estudio se desarrolló en la zona sur de la provincia de Córdoba, Argentina en un establecimiento privado de ciclo completo, ubicado geográficamente en el sur de la provincia de Córdoba, Pedanía Italó del Departamento General Roca, República Argentina. Situada a una altura $153.5 \mathrm{msnm}$, latitud $34^{\circ} 31^{\prime} 8$ " sur, longitud $63^{\circ} 56^{\prime} 39^{\prime \prime}$ oeste. El clima es templado, tipo continental con inviernos frescos y veranos cálidos,
Recientemente ( $\mathrm{Du}$ et al., 2017; Greenwood et al., 2017) determinaron diferentes alteraciones metabólicas, y de composición corporal, en diferentes puntos de la curva de crecimiento postnatal como producto del mecanismo de programación fetal. Para Caton et al. (2018) los mismos podrían estar relacionados con cambios producidos sobre los requerimientos energéticos de la progenie.

En la región pampeana Argentina la alimentación preparto en bovinos de cría, generalmente, se realiza con recursos de mediano a bajo nivel proteico como por ejemplo, pastizales naturales con especies principalmente estivales, pasto llorón (Eragrostis curvula), digitaria (Digitaria eriantra) diferidos o rastrojos de maíz (Zea mays) entre otras. Sin embargo, existen excepciones, por ejemplo, en sistemas comerciales de alto nivel de producción que venden parte de su producción como recurso genético, en núcleos genéticos o, eventualmente, en sistemas comerciales cuando existen excedentes de forrajes de buena calidad como avena (Avena sativa), triticale (Tritico secale), centeno (Cereale secale), y alfalfa (Medicago sativa) (Canosa, 2003; Moralejo, 2004; Arelovich, et al., 2008;.Faverin y Machado, 2019). La evaluación nutricional de pasturas no es una práctica común en sistemas de cría, y en general se desconoce, en detalle, su nivel de aporte proteico. El objetivo del presente trabajo fue evaluar el efecto de una dieta pastoril preparto con elevado nivel proteico Triticale (Tritico secale) sobre el crecimiento de la progenie, en vacas de cría durante el último tercio de la gestación.

con una temperatura promedio de $30{ }^{\circ} \mathrm{C}$ y una mínima de $10{ }^{\circ} \mathrm{C}$. Está situado en el distrito fitogeográfico del "Espinal" que se caracteriza por especies como espinillo (Acacia caven), que es la predominante, cedrón (Aloysia citrodora), poleo (Mentha pulegium), algarrobo blanco (Prosopis alba) y negro (Prosopis nigra) (Dimitri et al., 1999 ; 2000).

\section{Tratamientos}

Las hembras fueron sometidas al mismo manejo nutricional desde 2014, habiendo gestado y amamantado un ternero por año, desde esa fecha hasta el momento de la realización del ensayo (2016), al que ingresaron sin ternero al pie. En el período preexperimental se les administró una pastura de alfalfa (Medicago sativa) hasta los 6 meses de gestación (de enero a junio). Luego, durante el último 
tercio de la gestación (julio, agosto y septiembre) se dividieron las hembras en dos grupos de alimentación en pastoreo, sobre los que permanecieron hasta el parto: triticale (Cereale cecale) (T) y una pastura natural degradada (PD) en la que se relevaron especies naturales principalmente compuesta por hierbas dicotiledóneas del género Baccharis: mío-mío (Baccharis coridifolia) y carqueja (Baccharis articulata), así como monocotiledóneas de la familia Poáceas como espartillo (Spartina sp.), pasto alambre
(Sporobolus sp.), flechilla (Stipa sp.) y cola de zorro (Setaria leucopila).

El análisis nutricional de ambos tratamientos realizado en el Laboratorio de Nutrición de la Universidad Nacional de Rio Cuarto se presenta en la Cuadro 1. El aporte de PB de cada tratamiento fue superior en $10 \%(\mathrm{PD})$ y $59 \%(\mathrm{~T})$ a los requerimientos diarios de las hembras gestantes en el último tercio de la gestación estimados en base NRC (2000) (Cuadro 2).

Cuadro 1 Valores nutricionales de Proteína Bruta (PB), Digestibilidad y Mcal EM/kg MS de los tratamientos Triticale (T) y Pastura Degradada (PD) .

\begin{tabular}{lccc}
\hline & PB (\%) & Digestibilidad (\%) & Mcal EM/kg MS \\
\hline Triticale & 18.20 & 68.70 & 2.48 \\
Pastura Degradada & 13.92 & 65.50 & 2.36 \\
\hline
\end{tabular}

Cuadro 2 Requerimientos de PB, Mcal EM estimados para las hembras gestantes en el último tercio de la gestación

\begin{tabular}{lcc}
\hline \multicolumn{2}{c}{ Requerimientos } \\
\hline & Triticale (T) & Pastura degradada (PD) \\
PB (g/día) & 958.35 & 958.35 \\
Mcal EM/día & 13.25 & 13.60 \\
Consumo (kg MS/día) & 8.37 & 7.75 \\
\hline
\end{tabular}

Durante el postparto la alimentación de ambos grupos fue sobre pastura de alfalfa (Medicago sativa) Grupo 7 (Reposo intermedio) cultivar Dekalb 170 hasta el destete. La composición promedio de la pastura de alfalfa utilizada en estado vegetativo fue de $22.77 \%$ de PB, $64 \%$ de digestibilidad y 2.53 Mcal $\mathrm{Mm} / \mathrm{kgMS}$, mientras que estos valores entre inicio de floración y floración completa fueron de $21.65 \%$ de $\mathrm{PB}, \quad 61.5 \%$ de digestibilidad in vitro $\mathrm{y}$ una concentración energética de $2.4 \mathrm{Mcal} \mathrm{EM} / \mathrm{kg}$ MS. El análisis de variables se realizó sobre la progenie nacida en 2017 de ambos grupos de vacas con alimentación diferenciada en el último tercio de la gestación. Durante el periodo de la evaluación las progenies de ambos grupos de vacas estuvieron expuestos a las mismas condiciones, siendo alimentados sobre pastura (Cuadro 3) con el acceso de todos animales en el mismo grupo.

\section{Resultados y Discusión}

Los resultados de los valores medios obtenidos en cada tratamiento y la comparación de medias para las variables analizadas: peso al nacer (PN), peso a los 205 días (P205), aumento diario de peso vivo predestete (ADPV) y peso al año (PA) se presentan en el Cuadro 4. Según los resultados observados en las condiciones de este ensayo, puede decirse que cuando el aporte de proteína preparto fue mayor, el potencial de

\section{Variables evaluadas}

Para evaluar los efectos sobre el crecimiento de las crías se determinó: peso al nacer (PN), peso ajustado a los 205 días (P205), aumento diario de peso vivo pre destete (ADPV) y peso al año (PA).

\section{Análisis estadístico}

Se empleó un diseño experimental completamente aleatorizado con dos repeticiones y se asignaron al azar 8 animales a cada tratamiento. Se sometieron los datos de las variables a análisis de varianza (ANOVA) con el programa estadístico R (R Development Core Team, 2009) para evaluar el efecto del nivel proteico en el último tercio de la gestación sobre el peso al nacer, peso corregido a los 205 días, peso al año. La prueba de Tukey fue utilizada para determinar diferencias significativas entre los tratamientos.

crecimiento de la progenie fue afectado negativamente, diferencias significativas ( $\mathrm{p}<0.05)$, sin afectar el peso al nacer. Respecto a P205 la diferencia fue de $38 \mathrm{~kg}$ entre los dos grupos, siendo superior para el grupo PD, con menor proporción de PB (13.92 \%).

Los resultados de PN concordaron con lo observado por otros autores, aun cuando utilizaron diferentes 
fuentes proteicas (Summers et al., 2012, Radunz et al., 2010; 2012, Larson et al., 2009, Wilson et al., 2015). Por otra parte, los resultados obtenidos en variables relacionadas al potencial de crecimiento post natal (P205, ADPV y PF), indicaron que un aporte superior a los requerimientos de $\mathrm{PB}$ en el último tercio de la gestación no se relacionaba necesariamente con mayores pesos de los terneros al destete, a diferencia de lo observado en otros trabajos (Summers et al., 2011; Radunz et al., 2010 ; 2012 ; Wilson et al., 2015) que utilizaron fuentes proteicas, con altas proporciones de proteína no degradable en rumen, a diferencia de lo que puede esperarse en una pastura de triticale (Tritico sereale). En concordancia a lo observado en PD y ADPV, Zago et al. (2019; 2020) concluyeron, a partir de un metaanálisis, que valores de $\mathrm{PB}$ o EM en niveles mayores a los recomendados por NRC en vacas preñadas durante el último tercio de la gestación reduce el peso de la progenie entre los 60 y los 205 días.

Summers et al. (2011) observó una mejor productividad, mayores ganancias y peso final, cuando sus madres consumieron alta proteína. Radunz et al., (2010; 2012) por su parte no observaron diferencias, al igual que Wilson et al. (2015) cuando se utilizó en las madres una dieta hiper proteica al $129 \%$ de los requerimientos de $\mathrm{PB}$ no observaron diferencias en P205, ADPV pre destete o en PF.

Cuadro 3. Alimentación y valores nutricionales post destete de la progenie para ambos tratamientos (T y PD).

\begin{tabular}{lccc}
\hline & Feb-Mar-Ab-May & Jun- Jul -Ag & Sept -Oct- Nov \\
\hline Forraje disponible & Alfalfa & Triticale & Alfalfa \\
PB (\%) & 21.5 & 19.5 & 18.7 \\
Digestibilidad (\%) & 67.9 & 67.3 & 61.5 \\
Mcal EM/ kg/MS & 2.34 & 2.42 & 2.21 \\
\hline
\end{tabular}

Cuadro 4 Medias, error estándar y comparación de medias para las variables pH, peso al nacer (PN), peso ajustado a los 205 días (P205), aumento diario de peso vivo pre destete (ADPV) y peso al año (PA).

\begin{tabular}{lccccc}
\hline \multicolumn{5}{c}{ Triticale (T) } & \multicolumn{2}{c}{ Pastura Degradada (PD) } & \\
\hline Variable & Media & Error Estándar & Media & Error Estándar & Probabilidad \\
\hline PN & 34 & 4.36 & 35 & 5.04 & $>0.05$ \\
P205 & 167.34 & 22.5 & 205.34 & 25.22 & $<0.05$ \\
ADPV & 0.65 & 0.09 & 0.83 & 0.11 & $<0.05$ \\
PA & 295.90 & 25.70 & 330.18 & 22.68 & $<0.05$ \\
\hline
\end{tabular}

Si bien en este ensayo no se evaluaron los aspectos metabólicos implicados en el proceso de ureagénesis, los resultados obtenidos pueden explicarse por los altos niveles de $\mathrm{PB}$, que se estima fueron principalmente proteína degradable en rumen, y pudieron generar mayor demanda metabólica sobre la vaca gestante por efecto de la ureagénesis excesiva.

Los forrajes de buena calidad aportan normalmente cantidades adecuadas de proteína degradable en rumen. Poseen alta disponibilidad ruminal, por lo cual son rápidamente degradadas y sirven fundamentalmente como fuente de $\mathrm{NH}_{4}^{+}$para la síntesis de proteína microbiana. Para ello es necesario que exista una fuente de hidratos de carbono de rápida disponibilidad. Es por ello que la producción ruminal de proteína puede verse afectada por desbalances entre la energía y proteína ruminales. Si el desequilibrio se debe a un exceso de nitrógeno, ya sea como proteína verdadera o como alguna fuente de NNP, aumentará la concentración ruminal de $\mathrm{NH}_{4}{ }^{+}$debido a que no es empleado para sintetizar proteínas bacterianas debido a la falta relativa de cadenas carbonadas (Hall y Huntington, 2008 ; Hersom, 2008).
Hare et al. (2018) evaluaron el uso de una dieta con exceso de proteínas metabolizable en rumen (133\% de los requerimientos) en la gestación tardía en ganado de carne sobre la concentración de urea plasmática que resultó mayor al tratamiento control (15.0 vs. 7.5 $\mathrm{mg} / \mathrm{dL})(\mathrm{p}<\mathrm{o.01}$ ), así como la fermentación ruminal y el balance de nitrógeno. El exceso de $\mathrm{NH}_{4}{ }^{+}$perjudica al animal en dos aspectos, por un lado, aumenta el pH ruminal y puede alterar su funcionamiento si éste supera el rango normal y por otro el $\mathrm{NH}_{4}{ }^{+}$es absorbido por el rumen y detoxificado en el hígado, mediante la formación de urea, con el consecuente incremento de urea plasmática y el gasto energético adicional para el rumiante (Bohnert et al., 2011).

La síntesis de $1 \mathrm{~mol}$ de urea requiere $1 \mathrm{~mol} \mathrm{de} \mathrm{NH}_{4}^{+} \mathrm{y}$ $1 \mathrm{~N}$ a-amino proveniente del aspartato. El costo energético de este proceso varía dependiendo del origen del $\mathrm{N}$, del balance de ATP y del NADH producido durante la regeneración de oxalacetato a partir del malato citosólico generado del fumarato liberado por el ciclo de la urea (Noro y Wittwer, 2017). La transformación de $1 \mathrm{~g}$ de $\mathrm{N}$ a urea requiere $7.3 \mathrm{kcal}$ (Deiros et al., 2004), lo que supone 1 Mcal de energía 
liberado por el ciclo de la urea (Noro y Wittwer, 2017). La transformación de $1 \mathrm{~g}$ de $\mathrm{N}$ a urea requiere $7.3 \mathrm{kcal}$ (Deiros et al., 2004), lo que supone $1 \mathrm{Mcal}$ de energía metabolizable (equivalente a $1.5 \mathrm{~L}$ de leche o pérdida de $200 \mathrm{~g}$ de grasa corporal al día) por cada $4 \mathrm{mg} / 100$ $\mathrm{mL}$ de aumento de niveles de $\mathrm{N}$ ureico en sangre o leche (Bach, 2004).

La ureagénesis (transformación del amonio excesivo en urea) está conectada al ciclo de Krebs y la Gluconeogénesis Hepática por metabolitos como aspartato, oxalacetato, cetoglutarato y glutamato. Elevadas concentraciones de amonio en rumen generan una excesiva demanda de intermediarios del ciclo de la urea, que provienen de precursores que son compartidos con el ciclo de Krebs y vía gluconeogénica. De esta manera se compromete la capacidad hepática ureagénica e interfiere negativamente sobre la gluconeogénesis hepática y el balance energético del animal (Noro y Wittwer, 2017). La hiperamonemia además está asociada a hiperglucemia producto de glucogenólisis hepática, con reducción de la utilización periférica de glucosa y una menor secreción pancreática de insulina (Noro y Wittwer, 2017).

En este contexto, puede inferirse que la energía disponible para los procesos de síntesis esté probablemente limitada y la gluconeogénesis disminuida con menores niveles de oxalacetato que conlleve a una menor disponibilidad de ATP (Hibbit, 1988). Conjuntamente existen evidencias que permiten relacionar el proceso de ureagénesis - cuando proviene de la detoxificación del amonio - con un mayor catabolismo del aminoácido metionina. Esta situación implica que, en rumiantes alimentados con altos contenidos de proteína degradable en rumen, pueden existir limitaciones para cubrir los requerimientos de este aminoácido y sus requerimientos podrían ser comparativamente más altos en estas condiciones de alimentación (Correa y Cuellar, 2004).

La consecuente competencia, de la ureagénesis, con otros procesos como gluconeogénesis, por la energía metabólica y diferentes metabolitos entre ellos la metionina, la disminución en su disponibilidad podría generar cambios epigéneticos en su genoma fetal y como resultado del mecanismo de programación fetal, afectar su potencial de crecimiento en comparación con aquellos individuos cuyas madres no fueron expuestas a alteraciones en su metabolismo durante esa etapa.

En humanos, se ha demostrado que la alimentación materna con una dieta rica en metionina, puede causar cambios epigenéticos y estos pueden transmitirse a la siguiente generación, lo que indique la herencia epigenética inducida por la dieta, en mamíferos es posible y que la metionina es uno de los factores que puede estar implicado en ellas (Morgan et al., 1999; Conney et al., 2001 Waterland, 2006; Sinclair et al., 2007). Jacometo et al. (2016) evaluaron el efecto de suplementar con metionina protegida durante las últimas 3 semanas de gestación en hembras Holstein y demostraron efectos importantes en el feto sobre funciones como gluconeogénesis y sensibilidad a insulina, lo que permite inferir que la disponibilidad de metionina en el feto durante la gestación tardía favorece el desarrollo de estas rutas metabólicas.

\section{Conclusiones}

En conclusión, en las condiciones de este ensayo, el aporte de una pastura de Triticale (Tritico secale) de elevado nivel proteico, $59 \%$ por encima de los requerimientos estimados según NRC (2000), durante el último tercio de la gestación estuvo relacionada a menores valores de peso al destete, aumento diario de peso vivo y peso al año de la progenie. Estos resultados contribuyen a remarcar la necesidad de verificar la alimentación de la vaca de cría, no solo en base a cubrir sus requerimientos, sino en proveer un adecuado equilibrio entre la energía y proteína ruminales, con el objetivo de prevenir los efectos desequilibrio metabólico sobre la productividad en conjunto del sistema de producción de carne.

\section{Literatura Citada}

Arelovich, H. M., R. D. Bravo, \& M. F. Martínez. (2011). Development, characteristics, and trends for beef cattle production in Argentina. Animal frontiers, 1(2), 37-45.

Bach, A. 2004. La reproducción del vacuno lechero: Nutrición y fisiología. XVII Curso de Especialización FEDNA. Purina España. http://www.etsia.upm.es/fedna/capitulos/2001CAP V.pdf.
Barker, D. J. P. 2004. Developmental origins of well being. Philos. Trans. Royal Soc. London 359:13591366.

Barker, D. J. P. Editor. 1992. Fetal and Infant Origins of Adult Disease. BMJ Publishing Group, London.

Bohnert, D. W., T., A. A. Del Curto, M. L. Clark, M. L., Clark, M. L., Merrill, S. J. Falck and D. L. Harmon. 2011. Protein supplementation of ruminants consuming low-quality cool- or warm-season forage: Differences in intake and digestibility. J. Anim. Sci. 89: 3707-3717. 
Canosa F. Evolución, potencial y limitantes de la cría en la Argentina. Cuaderno de Actualización Técnica en Cría Vacuna. CREA, 2003

Caton, J. S., M. S., Crouse, L. P., Reynolds, T. L Neville, C. R., Dahlen, A. K Ward,., \& K. C. Swanson, (2018). Maternal nutrition and programming of offspring energy requirements, Translational Animal Science, Volume 3, Issue 3, Pages 976-990, https://doi.org/10.1093/tas/txy127

Cooney C.A., A.A., Dave G.L. Wolff 2002 Maternal methyl supplements in mice affect epigenetic variation and DNA methylation of offspring. J Nutr; 132: 2393S-2400S.

Cooney, C. A., A. A. Dave, , and G. L. Wolff, 2001. Maternal methyl supplements in mice affect epigenetic variation and DNA methylation of offspring. J. Nutr. 132(Suppl. 8), 2393S-2400S.

Correa, H.J., y A.E. Cuellar 2004. Aspectos claves del ciclo de la urea con relación al metabolismo energético y proteico en vacas lactantes. Revista Colombiana de Ciencias Pecuarias 17(1): 29-38.

Currier, T.A D.W., Bohnert, S.J. Falck, y S.J. Bartle, 2004. Influence of energy supplementation on grazing ruminants: requirements and responses. J. Anim. Sci. 82:1508-1517

Deiros, J., L.A. A.I. Quintela, J.J. Peña, M. Becerra, , G. Barrio Alonso, 2004. Urea plasmática relación con el equilibrio energético y parámetros reproductivos en vacunos lecheros. Arch. Zootec. 53:141-151

Dimitri, M. J.; R. F. Leonardis, y J. Santos Biloni. 1999. El nuevo libro del árbol. Tomo I. Especies Forestales de la Argentina Occidental. $1^{0}$ Edición. Buenos Aires. El Ateneo. ISBN: 950-02-8467-7. 120 p.

Dimitri, M. J.; R. F. Leonardis, y Santos Biloni J. 2000. El nuevo libro del árbol. Tomo II. Especies Forestales de la Argentina Oriental. $3^{\circ}$ Edición. Buenos Aires. El Ateneo. ISBN: 950-02-8474-X. 124 p

Du M., J Tong., J Zhao., K.R. Underwood, M., Zhu S.P., Ford P.W., Nathanielsz 2010. Fetal Programming of Skeletal Muscle Development in Ruminant Animals. . J. Anim.Sci 88(13): 51-60 .

Du, M., S. P. Ford, and M. J. Zhu. 2017. Optimizing livestock production efficiency through maternal nutritional management and fetal developmental programming. Anim. Front. 7:5-11. doi: 10.2527/af.2017-0122.

Du M., J Tong., J Zhao., K.R. Underwood, M., Zhu S.P., Ford P.W., Nathanielsz . 2009. Fetal programming of skeletal muscle development in ruminant animals. J. Anim. Sci. 88.

Du, M.; X. Yan,; F Tong,; J. Zhao, and M. Zhu, 2010.Maternal obesity, inflammation, and fetal skeletal muscle development. Biol Reprod 82.
Faverin, C., \& C. Machado, (2019). Tipologías y caracterización de sistemas de cría bovina de la Pampa Deprimida. Chilean journal of agricultural \& animal sciences, 35(1), 3-13.

Ferguson J. and W. Chalupa, 1989. Impact of protein nutrition on reproduction in dairy cows. J. Dairy Sci. 72(3):746-66

Funston, R.; J. Martin,; D. Adams, and D. Larson, 2010. Winter grazing system and supplementation of beef cows during late gestation influence heifer progeny. J. Anim. Sci. 88.

Gicquel C , A. El-Osta , Y. Le Bouc 2008 Epigenetic regulation and fetal programming .Best Pract Res Clin Endocrinol Metab 22:1-16

Greenwood, P., E. Clayton, and A. Bell. 2017. Developmental programming and beef production Anim. Front. 7:38-47. doi:10.2527/af.2017-0127.

Hall M. B; G. B., Huntington., 2008, Nutrient synchrony: Sound in theory, elusive in practice, Journal of Animal Science, Volume 86, Issue suppl_14, Pages E287-E292, https://doi.org/10.2527/jas.2007-0516

Hare, K.S., K. M Wood, K. Acton C. Fitzsimmons G. and Penner C. 2018 Oversupplying metabolizable protein in late gestation for beef cattle: effects on prepartum BW, ruminal fermentation, nitrogen balance, and skeletal muscle catabolism . J. Anim. Sci 9713

Hersom M. J., 2008 Opportunities to enhance performance and efficiency through nutrient synchrony in forage-fed ruminants, Journal of Animal Science, Volume 86, Issue suppl_14, April, Pages https://doi.org/10.2527/jas.2007-0463

Hibbitt, K.G. 1984, Effect of protein on the health of dairy cows., Recent advances in animal nutrition. Butterworth \& Co. Publ., London W. Haresign, D.J.A. Cole (Eds.), UK, pp. 189-200

Jacometo, C, B., Z. Zhou, , D. Luchini, , E. Trevesi, , M, N. Correa, \& J, J. Loor, 2016. Maternal rumenprotected methionine supplementation and its effect on blood and liver biomarkers of energy metabolism, inflamation, and oxidative stress in neonatal Holstein calves. J. Dairy Sci., 99, 67536763.

Lan, X., E. C. Cretney, , J. Kropp, , K Khateeb,., M. A Berg, F. Peñagaricano, , R. Magness, , A. E. Radunz, and H. Khatib, 2013. Maternal Diet during Pregnancy Induces Gene Expression and DNA Methylation Changes in Fetal Tissues in Sheep. Front Genet.;4:49. 
Larson, D. M., J. L. Martin, D. C. Adams, and R. N. Funston. 2009. Winter grazing system and supplementation during late gestation influence performance of beef cows and steer progeny. J. Anim. Sci. Mar;87(3):1147-55. doi: 10.2527/jas.2008-1323

Martin, J.L., K.A. Vonnahme, D.C. Adams, G.P. Lardy, and R.N. Funston. 2007. Effect of dam nutrition on growth and reproductive performance of heifer calves. J. Anim. Sci. 85:841-847. doi:10.2527/jas.2006-337

McEvoy T.G., J.J Robinson., P.A Findley., R.P. Aitken and I.S. Robertson (1997. Dietary excesses of urea influence the viability and metabolism of preimplantation sheep embryos and may affect fetal growth among survivors. Anim Reprod. ;47(1-2):7190

Moralejo R. 2004 Alimentación y condición corporal de la vaca de cría en la zona semiárida Conferencia. Revista Angus, Bs. As. http://www.produccionanimal.com.ar/informacion_tecnica/cria_condicion _corporal/68-cc_y_alimentacion.pdf

Morgan HD, HG Sutherland, DI Martin, E.Whitelaw 1999 Epigenetic inheritance at the agouti locus in the mouse. Nat Genet; 23: 314-8.

Nathanielsz, P.W., L. Poston, , P.D. Taylor, (2007). In utero exposure to maternal obesity and diabetes: Animals models that identify and characterize implications for future health. Clinics Perinatology 34: 515-526.

Noro, M. y F. Wittwer, 2017. Hemoglobinuria posparto en vacas de tres rebaños lecheros de la región del Bío-Bío, Chile. Revista MVZ Córdoba. 16 (3): 27852792

NRC 2000. Nutrient Requirements of Beef Cattle. National Academy Press, (Washington D.C)

R Development Core Team. 2009. R : A language and environment for statistical computing. R Foundation for Statistical Computing, Vienna, Austria. ISBN 3900051-07-0, http://www.R-project.org.\}

Radunz, A. E. 2009. Effects of prepartum dam energy source on progeny growth, glucose tolerance, and carcass composition in beef and sheep. PhD Diss. The Ohio State. Univ., Columbus.

Radunz, A. E., F. L. Fluharty, A. E. Relling, T. L. Felix, L. M. Shoup, H. N. Zerby, and S. C. Loerch. 2012. Prepartum dietary energy source fed to beef cows: II. Effects on progeny postnatal growth, glucose tolerance, and carcass composition. J. Anim. Sci. 90:4962-4974.
Radunz, A. E., F. L. Fluharty, M. L. Day, H. N. Zerby, and S. C. Loerch. 2010. Prepartum dietary energy source fed to beef cows: I. Effects on pre- and postpartum cow performance. J. Anim. Sci. 88:27172728.

Reed, S.A., and K.E. Govoni. 2017. How mom's diet affects offspring growth and health through modified stem cell function. Anim. Front. 7(3):25-31.

Reynolds, L. P., \& J. S. Caton, (2012). Role of the preand post-natal environment in developmental programming of health and productivity. Molecular and Cellular Endocrinology, 1(354), 54-59.

Scheffler, J.; M. McCann,; S. Greiner,; H. Jiang,; M. Hanigan,; G. Bridges,; S. Lake, and D. Gerrard, 2014. Early metabolic imprinting events increase marbling scores in fed cattle. J Anim Sci., 92(1):3204 .

Sinclair, K. D., C. Allegrucci, R. Singh, , D. S Gardner, S. Sebastian, , J Bispham ... \& R. G Lea,. (2007). DNA methylation, insulin resistance, and blood pressure in offspring determined by maternal periconceptional $\mathrm{B}$ vitamin and methionine status. Proceedings of the National Academy of Sciences, 104(49), 19351-19356.

Summers A. F. and R. N. Funston. 2012. Fetal programming: implications for beef cattle production Beef Improvement Federation's Annual Meeting and Research Symposium. http://www.bifconference.com/bif2012/proceeding s-pdf/o7funston.pdf

Summers, A. F., K. H. Ramsay, and R. N. Funston. 2011. The effects of maternal nutrition on steer progeny performance. Prof. Anim. Sci. 27:251-256.

Underwood, K.; J. Tong ; P. Price,; A. Roberts,; E. Grings,; B. Hess,; W. Means, and M. Du, 2010. Nutrition during mid to late gestation affects growth, adipose tissue deposition, and tenderness in cross-bred beef steers. Meat Sci. 86.

Wilson, T. B., A. R. Schroeder, F. A. Ireland, D. B. Faulkner, and D.W. Shike. 2015. Effects of late gestation distillers grains supplimentatios on fallcalving beef cow performance and steer calf growth and carcass characteristics. J.Anim.Sci. 93(10):4843-51. doi: 10.2527/jas.2015-9228.

Zago, D., M. E. A Canozzi \& J. O. J. Barcellos, (2019). Pregnant cow nutrition and its effects on foetal weight-a meta-analysis. The Journal of Agricultural Science, 157(1), 83-95.

Zago, D. , M. E. A., Canozzi\& J. O. J. Barcellos, (2020). Pregnant beef cow's nutrition and its effects on postnatal weight and carcass quality of their progeny. Plos one, 15(8), eo237941. 